\title{
Photooxidation of Acyclovir with Thermally Generated Triplet Excited Ketones. A Comparison with Type I and II Photosensitizers
}

\author{
Jawaid Iqbal,* Adil Husain, and Anamika Gupta \\ Department of Chemistry, Organic Chemistry Section, Aligarh Muslim University; Aligarh 202002 (U.P) India.
}

Received November 18, 2005; accepted January 7, 2006

\begin{abstract}
The antiviral drug acyclovir (Ac, 1) was treated with triplet excited ketones, which have been generated in thermal decomposition of 3-(hydroxymethyl)-3,4,4-trimethyl-1,2-dioxetane (HTMD), in the dark. Three major oxidation products were detected by means of spectroscopic measurements. The products were (2-hydroxyethoxy) methyl spiroiminodihydantoin (2), (2-hydroxyethoxy) methyl (amino)-2-imino-1,2-dihydroimidazole-5one (3), and 2,2-diamino-4-[(2-hydroxyethoxy) methyl) amino)-5-[2H]-oxazolone (4). Equal amounts of type I and type II photooxidation products were found, as could be established by comparison with predominant type I (riboflavin) and type II (rose bengal) photosensitizers. The concentration and time profiles for the HTMDinduced oxidation of Ac were also determined. The participation of singlet oxygen in HTMD-induced oxidation was confirmed by the substantial $D_{2} O$ effect in the formation of spiroiminodihydantoin (2).
\end{abstract}

Key words acyclovir; photooxidation; 1,2-dioxetane; triplet-excited ketone

Over the last decade there has been increasing interest in the photosensitization mechanism in the biological systems, in relation to both deleterious and therapeutic aspects of this phenomenon. Photosensitization reactions are generally considered as belonging to either the type I (radical mediated) or type II (singlet oxygen mediated). ${ }^{1)}$ Many of the endogenous (e.g. flavins, tetrapyrrols, protoporphyrins etc.) or exogenous photosensitizers can elicit phototoxic or photoallergic responses. $^{2,3}$ Likewise there are many sensitizing dyes e.g. rose bengal, benzophenone, riboflavin etc. that are activated by light.

An important class of photooxidative sensitizers constitutes the triplet-excited ketones, ${ }^{4,5)}$ which are of biological interest since they may be generated in cellular systems upon exposure of endogenous chromophores to the UV irradiation or by dark reactions (e.g. lipid peroxidation and enzymatic oxidation). ${ }^{6-8)}$ Triplet excited ketones may be produced by thermal decomposition of 1,2-dioxetanes, alternative to their conventional photochemical generations. ${ }^{9,10)}$ These thermally generated triplet-excited ketones are analogous to those produced photochemically and operate as type I or type II photooxidants. $^{11)}$ 1,2-Dioxetanes are unique class of four membered ring peroxides and are of biological interest, since they have been implicated as labile intermediates in oxidative stress. $^{5)}$

Acyclovir (9-[(2-hydroxyrthoxy) methyl) guanine) (Ac, 1) is an antiviral drug used for the treatment of herpes encephalitis caused by herpes simplex virus or varicella zoster virus infections. In our recent study for the singlet oxygen mediated photooxidation of Ac in aqueous solution, ${ }^{12}$ we have isolated spiroiminodihydantoin (Sp, 2), imidazolone $(\mathbf{I m}, \mathbf{3})$ and oxazolone $(\mathbf{O x}, 4)$ as the three major products (Chart 1). Herein we have investigated photooxidation of Ac in presence of thermally generated triplet excited ketones. The distribution of products were compared with those of Ac photooxidation by a type I sensitizer, riboflavin and a type II sensitizer rose bengal. ${ }^{13,14)}$

\section{Experimental}

Chemicals and Reagents All chemicals used were of analytical grade. Acyclovir was extracted from the commercial medicament Acivir (Cipla
Limited, Mumbai, India) with a soxhlet extractor, purified by TLC and recrystallized from the same solvent. Melting point, ${ }^{1} \mathrm{H}-\mathrm{NMR}$ and co-TLC with the authentic pure sample determined the purity of acyclovir. HTMD was synthesized according to the literature procedure. ${ }^{15)}$ Standard samples of Spiroiminodyhydantoin, imidazolone and oxazolone were prepared by rose bengal photosensitized oxidation of Ac.

Oxidation Procedure For the dioxetane mediated oxidation of Ac, a $0.5 \mathrm{~mm}$ solution of $\mathbf{A c}$ in $10 \mathrm{~mm}$ sodium cacodylate buffer ( $\mathrm{pH} 7.0)$ and 10 vol $\%$ dioxetane solution in acetonitrile was kept at $50{ }^{\circ} \mathrm{C}$ for several hours in absence of light. The photosensitized oxidation of phosphate buffered solution of Ac $(0.5 \mathrm{~mm})$ was carried out in the presence of photosensitizers riboflavin or rose bengal. A $150 \mathrm{~W}$ sodium lamp was used for the carrying out photosensitized oxidations of Ac with riboflavin and rose bengal. The lamp was placed at $15 \mathrm{~cm}$ below the bottom of an ice filled beaker, in which a round bottom flask having reaction mixture was placed. HTMD-induced photooxidation was carried out in dark for $18 \mathrm{~h}$ at $50^{\circ} \mathrm{C}$ by using acetonitrile ( $10 \%$ by vol) as co-solvent while the photooxidation by riboflavin and rose bengal was carried out for $2.5 \mathrm{~h}$. A number of products were indicated on TLC at the end of the reaction, from which only three major photoproducts $\mathbf{2}, \mathbf{3}$ and $\mathbf{4}$ were obtained in isolable yields. After the given time period the amount of photoproducts formed and un-oxidized Ac was assessed by isolation and purification of the photolysate using silica gel column chromatography.

Relative yields of products were determined based on the consumed Ac and the mean value of at least 3 independent runs was reported (Table 1). The concentration and time profile for HTMD induced oxidation of Ac $(0.5 \mathrm{~mm})$ at $50^{\circ} \mathrm{C}$ were determined by using $10 \mathrm{~mm}$ sodium cacodylate buffer $(\mathrm{pH} 7)$ as a reaction medium with $10 \mathrm{vol} \%$ acetonitrile as co-solvent. Effects of $\mathrm{D}_{2} \mathrm{O}$ on the yield of $\mathbf{S p}$ in HTMD-induced photooxidation were also observed to confirm the involvement of singlet oxygen.

\section{Results and Discussion}

On the thermal treatment of Ac with HTMD at $50{ }^{\circ} \mathrm{C}$ three major products were identified. The products were (2-hydroxyethoxy) methyl spiroiminodihydantoin (2), (2-hydroxyethoxy) methyl (amino)-2-imino-1,2-diydroimidazole-

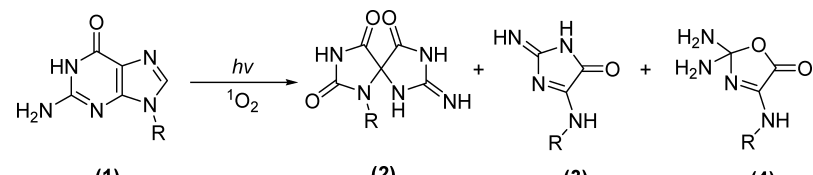

(1)

(2)

(3)

(4)

$\mathrm{R}=八 \mathrm{O} \mathrm{OH}$ 
5-one (3), and 2,2-diamino-4-[(2-hydroxyethoxy) methyl)amino)-5-[2H]-oxazolone (4) (Chart 2). All the photoproducts were identified by comparing their spectral data and TLC with those of authentic pure samples of spiroiminodihydantoin (Sp), imidazolone (Im) and oxazolone (Ox) generated in the rose bengal mediated photooxidation of Ac. The spectral data of all the three products were found to be in close agreement with the well-established singlet oxygen mediated Ac photooxidation products ${ }^{12)}$ (Chart 1). Similar products pattern was obtained when Ac was exposed in aerated aqueous solution of photoexcited riboflavin and rose bengal. Different yields of products were obtained depending upon the photosensitizer, which was used. In the type II sensitized photooxidation by rose bengal, spiroiminodihydantoin was detected as the major product whereas the type I photosensitizer riboflavin gave imidazolone and oxazolone as major product. For HTMD, which entails both photooxidation modes, the yields of type I and type II products were in close agreement. The $\mathbf{S p} /(\mathbf{I m}+\mathbf{O x})$ product ratio may serve as mechanistic probe to access the predominant photooxidation products in type I and type II photosensitized oxidative modifications. For example in riboflavin sensitized photooxidation the product ratio lies below 1 while for rose bengal sensitized oxidation it is above 3. For HTMD the product ratio is 1.3. Also for the product balance, the HTMD oxidation lies between the type I (40\%, entries 2$)$ and type II (57\%, entries 3 ) process. Furthermore the relative yield of $\mathbf{S p}$ in HTMDinduced oxidation $(30 \%)$ is significantly higher $(15 \%)$ than that of riboflavin sensitized oxidation and smaller $(15 \%)$ than

Table 1. Product Balance of Ac Oxidation by Dioxetane HTMD and by Riboflavin and rose bengal Photosensitizers

\begin{tabular}{|c|c|c|c|c|c|c|c|}
\hline \multirow{3}{*}{ Entry } & \multirow{3}{*}{$\begin{array}{c}\text { Concentration } \\
\left(\mu_{\mathrm{M}}\right)\end{array}$} & \multirow{3}{*}{ Oxidant } & \multicolumn{4}{|c|}{ Product yields $(\%)^{a)}$} & \multirow{3}{*}{$\begin{array}{l}\text { Product } \\
\text { balance }\end{array}$} \\
\hline & & & \multirow{2}{*}{$\frac{\text { Type II }}{\text { Sp }}$} & \multicolumn{3}{|c|}{ Type I } & \\
\hline & & & & Im & $\mathbf{O x}$ & Total & \\
\hline 1 & 10000 & $\mathrm{HTMD} / 50^{\circ} \mathrm{C}$ & $30 \pm 3$ & $8 \pm 2$ & $15 \pm 1$ & $23 \pm 2$ & $53 \pm 3$ \\
\hline 2 & 10 & Riboflavin & $15 \pm 2$ & $7 \pm 1$ & $18 \pm 2$ & $25 \pm 3$ & $40 \pm 3$ \\
\hline 3 & 10 & Rose bengal & $45 \pm 1$ & $5 \pm 1$ & $7 \pm 1$ & $12 \pm 2$ & $57 \pm 2$ \\
\hline
\end{tabular}

a) Relative yields based on consumed Ac, mean value of three independent runs. that in the rose bengal sensitization. In contrast the yields of the type I products in HTMD-induced oxidation (23\%) are comparable with those of type I sensitizer (25\%) and significantly higher than those in type II photooxidation (12\%).

This difference in products distribution may be accounted for the fact that rose bengal produces singlet oxygen in large amount by a type II mechanism whereas riboflavin, which act mainly by type I photosensitized oxidation do not produce significant amount of singlet oxygen. On the other hand HTMD, is neither a typical type I nor a characteristic type II photooxidant; in particular both photooxidation modes occur quite efficiently.

As shown in Fig. 1, when Ac was thermally treated with HTMD at $50{ }^{\circ} \mathrm{C}$ for $15 \mathrm{~h}$, a linearly dependent degradation of Ac was observed with increasing HTMD concentration. The absolute yield, based on initial amount of Ac, of characteristic type I photooxidation products, Ox and Im was $15 \%$, while type II product Sp was formed in up to $22 \%$ absolute yield at $25 \mathrm{~mm}$ HTMD concentration. In these reactions the sum of quantified products, relative to consumed Ac, amounted to be $56 \pm 4 \%$ and was independent of the HTMD concentration. Figure 2 shows the time profile for thermally HTMD induced photooxidation of Ac, which revealed a gradual increase of all oxidation products with time and product balance of $53 \pm 3 \%$ at $25 \mathrm{~mm}$ HTMD. Also in this case the product balance was independent of the reaction time.

The linear increase of Ac conversion with increasing HTMD concentration suggests that the HTMD oxidation of Ac is directly proportional to the amount of triplet-excited ketones formed by the thermal decomposition of the dioxetane. The time profile for photooxidation of Ac exhibited a decrease of Ac concentration with time. This reflects that the Ac is consumed in parallel with the generation of triplet-excited ketones.

Singlet oxygen quenchers such as DABCO, sodium azide etc. cannot be used to confirm the involvement of singlet oxygen in HTMD mediated photooxidation because they react with dioxetane. ${ }^{16,17)}$ Proof for the involvement of singlet oxygen in HTMD-induced oxidation comes from the substantial effect of $\mathrm{D}_{2} \mathrm{O}$ on the formation of $\mathbf{S p}$. Higher yields of $\mathbf{S p}$ in $\mathrm{D}_{2} \mathrm{O}$ compared to those of $\mathrm{H}_{2} \mathrm{O}$ implicate that singlet

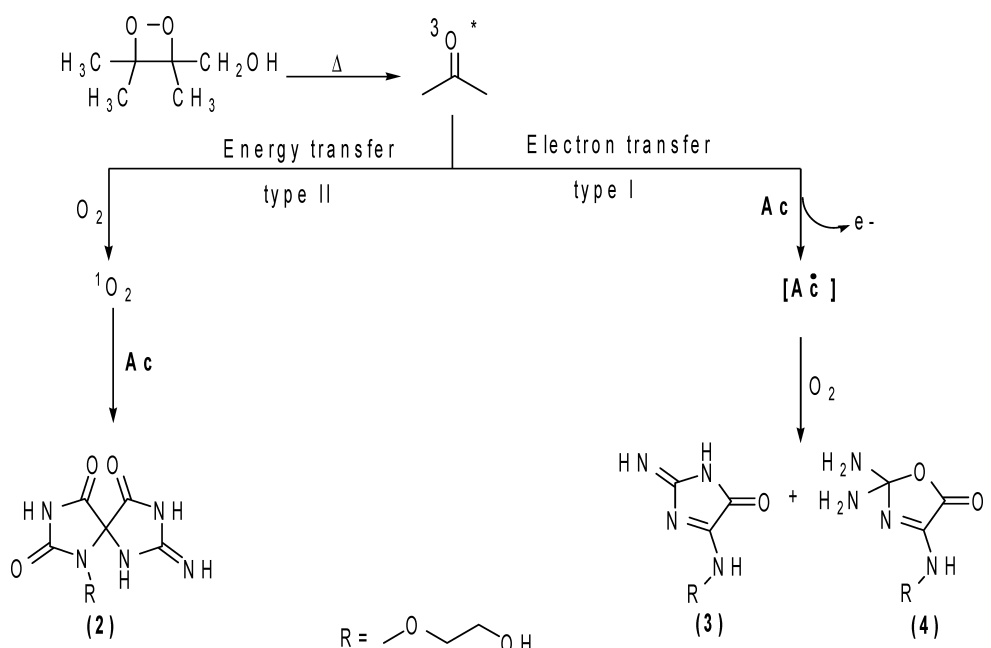

Chart 2 


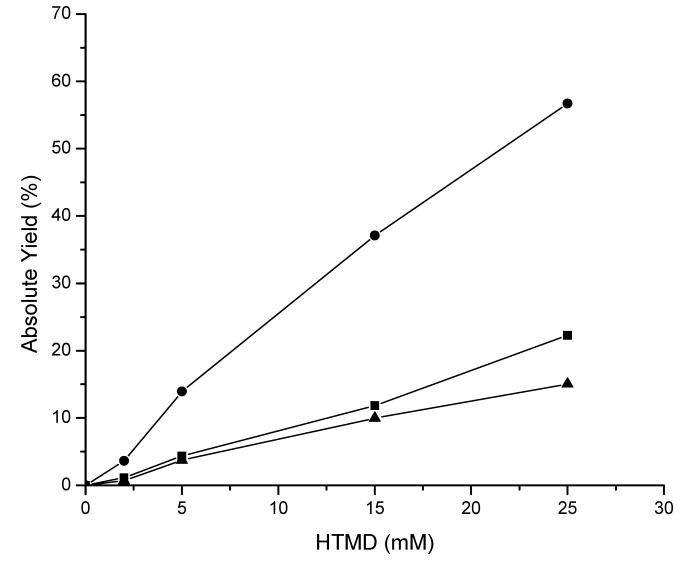

Fig. 1. Concentration Profile for the Thermally HTMD-Induced Photooxidation of Ac, Yields Derived from the Mean Values of Three Independent Runs, $(\bullet)$ Conversion Ac, $(\boldsymbol{\Delta})$ Yields of $\mathbf{O x}$ and $\mathbf{I m},(\mathbf{\square})$ Yields of Sp

oxygen is involved in HTMD mediated photooxidation.

Our present investigation reveals that triplet excited ketones generated in the thermal decomposition of HTMD oxidize Ac efficiently to the $\mathbf{S p}$, by a type II photooxidation mechanism and to the $\mathbf{O x}$ and $\mathbf{I m}$ by a type I mechanism. In addition, in the riboflavin sensitized type I oxidative modification of Acyclovir $\mathbf{O x}$ and Im was obtained as major product whereas $\mathbf{S p}$ was characterized as a major type II photooxidation product of rose bengal sensitized oxidation of Ac.

The investigation of photochemical properties of compounds used in clinical medicines is of great relevance from photobiological as well as photomedical point of view. Since singlet oxygen formation and the ensuing photooxidation of the drug and biomolecules is one of the main routes for the drug phototoxicity, the present findings may have an implication to the phototoxic effect of the drug.

\section{References}

1) Foote C. S., Photochem. Photobiol., 54, 659 (1991).

2) Tyrrell R. M., Mol. Aspects Med., 15, 1-77 (1994).

3) Dabestani R., Sik R. H., Davis D. G., Dubay G., Chignell C. F., Pho-

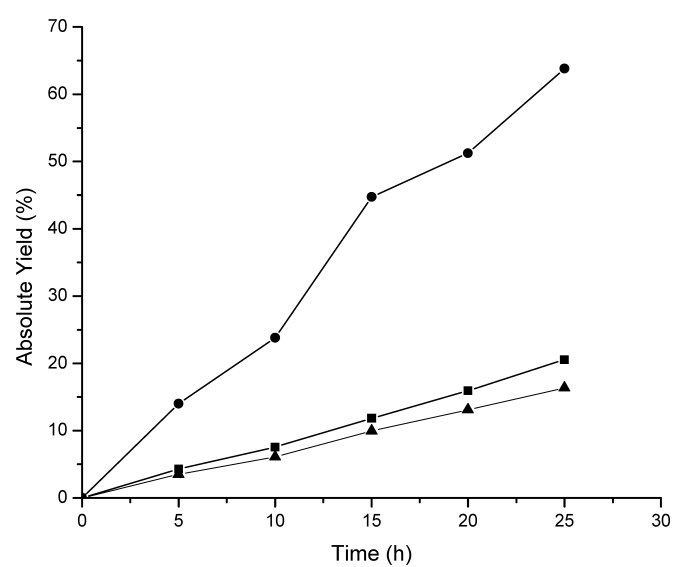

Fig. 2. Time Profile for the Thermally HTMD-Induced Photooxidation of Ac, Yields Derived from the Mean Values of Three Independent Runs, ( Conversion Ac, $(\Delta)$ Yields of $\mathbf{O x}$ and $\mathbf{I m},(\square)$ Yields of $\mathbf{S p}$

tochem. Photobiol., 58, 367-373 (1993).

4) Olsson T., Ekwall K., Ruusala T., Nucl. Acids Res., 21, 855-861 (1993).

5) Sies H., "Oxidative Stress," Oxidants and Antioxidants, Academic Press, New York, 1991.

6) Cilento G., Pure Appl. Chem., 56, 1179-1190 (1984).

7) Cadenas E., Photochem. Photobiol., 40, 823-830 (1984).

8) Baader W. J., Bohne C., Cilento G., Dunford H. B., J. Biol. Chem., 260, 10217-10225 (1985)

9) Adam W., Cilento G., Angew. Chem., Int. Ed. Engl., 22, 529-542 (1983).

10) Cilento G., Adam W., Photochem. Photobiol., 48, 361-368 (1988).

11) Adam W., Saha-Moller C. R., Schonberger A., J. Am. Chem. Soc., 118, 9233-9238 (1996).

12) Iqbal J., Husain A., Gupta A., Pharmazie, 60, 574-576 (2005).

13) Cadet J., Deccaroz C., Wang S. Y., Midden W. R., Isr. J. Chem., 23, $420-429$ (1983)

14) Buchko G. W., Cadet J., Can. J. Chem., 70, 1827-1832 (1992).

15) Leclercq D., Bats J.-P., Picard P., Moulines J., Synthesis, 9, 778-779 (1982).

16) Adam W., Heil M., J. Am. Chem. Soc., 114, 5591—5598 (1992).

17) Sundquist A. R., Hanusch M., Stahl W., Sies H., Photochem. Photobiol., 57, 785-791 (1993). 